\title{
FATORES CRÍTICOS DA TRANSFERÊNCIA DE CONHECIMENTO ENTRE UNIVERSIDADE E EMPRESA (U-E) ${ }^{1}$
}

\author{
Daniela Martins Diniz ${ }^{2}$ \\ Marina de Almeida Cruz ${ }^{3}$ \\ Victor Silva Correa ${ }^{4}$
}

http://dx.doi.org/10.1590/1413-2311.210.83919

\section{RESUMO}

O objetivo da pesquisa foi o de identificar os fatores críticos da transferência de conhecimento (TC) entre uma universidade pública brasileira e suas empresas parceiras e analisar como eles influenciaram no desempenho do processo. A revisão teórica contemplou a discussão sobre os fatores que, de acordo com a literatura na área, influenciam de forma decisiva na TC entre U-E. Em termos metodológicos, foi realizada uma pesquisa qualitativa com base no método de estudo de caso instrumental, sendo analisados dois projetos desenvolvidos entre uma universidade pública brasileira e suas empresas parceiras a partir de 14 entrevistas e documentos secundários. Os resultados revelam que dois fatores foram decisivos para a TC: o relacionamento estabelecido entre a universidade e empresas parceiras, e as características do professor (o seu know-how e a sua motivação para a TC). Enquanto o relacionamento contribuiu para minimizar a percepção de distância cultural entre U-E, o know-how dos professores tornou a parceria atrativa sob a ótica da empresa.

Palavras-chave: Transferência de conhecimento. Interação universidade-empresa. Fatores críticos da TC.

\footnotetext{
${ }^{1}$ Recebido em 15/06/2018, aceito para publicação em 27/07/2018.

${ }^{2}$ Universidade Federal de São João del Rei (São João del Rei, MG, Brasil) - danidiniz09@yahoo.com.br

${ }^{3}$ Fundação Dom Cabral (Belo Horizonte, MG, Brasil) - marina.almeida.cruz@gmail.com

${ }^{4}$ Universidade Paulista (São Paulo, SP, Brasil) - victorsilvacorrea@yahoo.com.br
} 


\title{
CRITICAL FACTORS OF KNOWLEDGE TRANSFER BETWEEN UNIVERSITY AND ENTERPRISE
}

\begin{abstract}
The objective of the research was to identify the critical factors of knowledge transfer (KT) between a Brazilian Public University and partner companies and to analyze how they influenced the process performance. The theoretical review included a discussion about the factors that, according to the literature in the area, decisively influence the CT between U-E. In methodological terms, a qualitative research was carried out based on the instrumental case study method, and two projects developed between the Brazilian Public University and partner companies were analyzed from 14 interviews and secondary documents. The results show that two factors were decisive for KT: the relationship established between the Brazilian Public University and partner companies, and the characteristics of the teacher (his know-how and his motivation. While the relationship contributed to minimize the perception of cultural distance between U-E, the know-how of the teachers made the partnership attractive from the perspective of the company.
\end{abstract}

Keywords: Knowledge transfer. university-company interaction. critical factors of KT.

\section{FACTORES CRÍTICOS DE LA TRANSFERENCIA DE CONOCIMIENTO ENTRE UNIVERSIDAD Y EMPRESA}

\section{RESUMEN}

El objetivo de la investigación fue identificar los factores críticos de la transferencia de conocimiento (TC) entre una universidad pública brasileña y las empresas asociadas y analizar cómo influenciaron en el desempeño del proceso. La revisión teórica contempló discusión sobre los factores que, de acuerdo con la literatura en el área, influencian de forma decisiva en la TC entre U-E. En términos metodológicos, se realizó una investigación cualitativa con base en el método de estudio de caso instrumental, siendo analizados dos proyectos desarrollados entre la universidad pública brasileña y las empresas asociadas a partir de 14 entrevistas y documentos secundarios. Los resultados revelan que dos factores 
fueron decisivos para la TC: la relación establecida entre la universidad pública brasileña y las empresas asociadas, y las características del profesor (su know-how y su motivación para la TC). Mientras que la relación contribuyó a minimizar la percepción de distancia cultural entre U-E, el know-how de los profesores hizo la asociación atractiva bajo la óptica de la empresa.

Palabras-clave: Transferencia de conocimiento. interacción universidad-empresa. factores críticos de la transferencia de conocimiento.

\section{INTRODUÇÃO}

O interesse na investigação da "transferência de conhecimento entre universidadeempresa" aumentou consideravelmente na década de 2000, se configurando como um importante tópico de pesquisa na atualidade (PERKMANN et al., 2013; FELIU; RODRÍGUES, 2017). Mudanças ambientais enfrentadas por universidades e empresas sinalizam que a interação entre o ambiente acadêmico e o empresarial se tornou estratégia vantajosa sob a perspectiva das duas partes, na medida em que representa uma fonte importante de recursos para a pesquisa científica e inovação para as empresas (FELIU; RODRÍGUES, 2017; ARAUJO, 2017). Algumas dessas mudanças estão relacionadas ao reconhecimento, pelas empresas, da necessidade de cooperar com atores externos para desenvolver inovação com menor custo e incerteza (CHESBROUGH, 2017) e, do lado da universidade, ao crescente discurso em torno da noção de "universidade empreendedora", que considera a transferência tecnológica uma das funções centrais da universidade, além do ensino e da pesquisa (ETZKOWITZ; LEYDESDORFF, 1997; MURMANN, 2013; FELIU; RODRÍGUES, 2017).

No Brasil, particularmente, a TC entre U-E tem ganhado espaço nas agendas de pesquisa a partir da promulgação, pelo governo federal, de dois marcos legais relacionados à inovação, especificamente, a Lei 10.973 de 2004 e a Lei 13.243 de 2016. A Lei de Inovação de 2004 determina que as instituições científicas instaladas no país criem um Núcleo de Inovação Tecnológica, que, dentre outras atribuições, devem cuidar do processo de TC para o setor produtivo. Em 2016, o governo promulgou a Lei 13.243, conhecida como o novo marco legal da inovação, representando uma tentativa de flexibilizar as atividades de inovação e de pesquisa desenvolvidas no país (ARAUJO, 2017; BRASIL, 2017). Portanto, a criação desses 
marcos representa um passo importante para o fortalecimento da TC entre o ambiente acadêmico e o empresarial no Brasil, chamando a atenção para a relevância de se investigar esse tópico de pesquisa.

Embora haja um reconhecimento sobre a importância da TC entre U-E na atualidade, verifica-se que esse processo é marcado por inúmeros desafios, indicando a necessidade de uma melhor compreensão dos fatores que influenciam no seu desempenho. As dificuldades de uma colaboração efetiva entre U-E são apontadas por diversos estudos, os quais revelam que apenas pequena parte do conhecimento produzido pelas universidades é aproveitada pelas empresas (IPIRANGA; FRETAS; PAIVA, 2010; ARAUJO, 2017).

Um dos motivos que pode explicar essa situação são as diferentes culturas do ambiente acadêmico e do empresarial. Diferenças relativas à missão de cada instituição (papel social, educacional e científico das universidades versus finalidade lucrativa das empresas); ao horizonte de tempo (perspectiva de médio/longo prazo da pesquisa científica versus lógica de curto prazo do setor empresarial); e ao foco da pesquisa (pesquisa básica/pura versus pesquisa aplicada), por exemplo, representam alguns obstáculos à interação entre U-E (BARBOLLA; CORREDERA, 2009; SCHOFIELD, 2013). No contexto brasileiro, especificamente, não há uma cultura de colaboração entre as instituições científicas e o setor empresarial no Brasil, o que é um fenômeno mais comum em países desenvolvidos (GARNICA; TORKOMIAN, 2009; DIAS; PORTO, 2014; STAL; FUJINO, 2016).

Diante do exposto, observa-se que há desafios quando se pensa em processos bemsucedidos de TC entre U-E, apontando para a importância de novas pesquisas que examinem os fatores que influenciam esse fenômeno. Nessa direção, o objetivo da pesquisa foi o de identificar os fatores críticos da transferência de conhecimento entre uma universidade pública brasileira e suas empresas parceiras, e analisar como eles influenciaram no desempenho do processo.

Em relação à relevância teórica do estudo, além de abordar um relacionamento pouco enfatizado na literatura sobre transferência de conhecimento (a díade universidade-empresa), a pesquisa buscou avançar na discussão dos fatores considerados críticos para o desempenho da transferência de conhecimento entre U-E no contexto de universidade brasileira, trazendo para a literatura insights relacionados à TC em países em desenvolvimento. Em termos de sua relevância prática, a pesquisa é importante ao fornecer insights para o aprimoramento da TC entre o ambiente acadêmico e o empresarial no país. 


\section{REFERENCIAL TEÓRICO}

\subsection{FATORES CRÍTICOS DA TC ENTRE U-E}

Neste estudo, a transferência de conhecimento entre U-E é definida como um processo relacional por meio do qual conhecimentos são desenvolvidos, compartilhados e aplicados com a finalidade de gerar resultados para as organizações envolvidas, que podem ser desde outputs acadêmicos até inovações em produto/processos/ferramentas. O interesse em torno dessa temática ganhou novos contornos na década de 2000 e a partir daí estudos foram desenvolvidos buscando-se compreender diversos aspectos relacionados a esse processo.

Dentre as várias possibilidades de recorte, o estudo realizado teve como foco os fatores que influenciam na TC entre U-E. Nesse sentido, buscou-se mapear, na literatura existente, os fatores que são mais reiterados e considerados decisivos para o desempenho de TC entre U-E. A identificação desses fatores ocorreu em duas etapas: a) primeiramente foram analisados trabalhos nacionais que contemplam discussão sobre fatores que influenciam na TC entre U-E (SEGATTO-MENDES; MENDES, 2006; GARNICA; TORKOMIAN, 2009; IPIRANGA; FRETAS; PAIVA. 2010; CLOSS et al. 2012; DESIDÉRIO; ZILBER, 2014; SOUZA; SANTOYO, 2016); b) na sequência, foram analisados estudos internacionais com o mesmo foco (SIEGEL et al., 2004; SANTORO; BIERLY, 2006; SHERWOOD; COVIN, 2008; BARBOLLA; CORREDERA, 2009; SCHOFIELD, 2013; ARAÚJO; TEIXEIRA, 2014).

Parte-se da premissa de que quanto mais determinados fatores forem reforçados em diferentes estudos, mais relevantes eles são para explicar o desempenho da TC entre U-E. Reunindo as contribuições dos estudos avaliados, observa-se que alguns fatores são reforçados por diferentes autores, quais sejam: a) relacionamento; b) condições universitárias; c) diferenças culturais; d) características dos professores.

Com relação ao primeiro fator, estudos na área apontam que a TC interorganizacional envolve necessariamente a conexão social entre, no mínimo, duas organizações, o que requer que as partes se relacionem para a troca de conhecimentos. Portanto, o relacionamento é um fator que deve ser investigado quando se busca compreender os fatores críticos para o desempenho da transferência (BARBOLLA; CORREDERA, 2009; CLOSS et al. 2012; ARAÚJO; TEIXEIRA, 2014). Resultados de pesquisas que analisam a interação entre U-E indicam que um relacionamento próximo, baseado em cooperação e confiança é um fator que favorece a TC, na medida em que: (a) minimiza a percepção de distância cultural entre o 
ambiente acadêmico e o empresarial, alinhando interesses na parceria (BARBOLLA; CORREDERA, 2009); (b) intensifica os vínculos sociais entre professores e funcionários da empresa ampliando o comprometimento das partes com a transferência (SIEGEL et al., 2004); (c) possibilita às duas partes se envolverem nas diferentes etapas do processo, facilitando a compreensão do conhecimento científico pelos funcionários da empresa (SHERWOOD; COVIN, 2008); (d) minimiza as diferenças de linguagem entre professores e funcionários da empresa (SEGATTO-MENDES; MENDES, 2006; CLOSS et al. 2012; 2013).

Particularmente no Brasil, o estudo de Garnica e Torkomian (2009) apontou que o baixo nível de comunicação entre a Universidade Estadual Paulista (UNESP) com uma empresa parceira e um acompanhamento pouco sistemático dos pesquisadores na fase de aplicação da tecnologia foram considerados um dos desafios enfrentados no processo de TC. Por outro lado, um bom nível de confiança da empresa no pesquisador "inventor" da tecnologia foi percebido como um fator que favoreceu o processo ao ampliar a disposição da empresa na parceria. Outro caso investigado por Garnica e Torkomian (2009), envolvendo a Universidade Federal de São Paulo, aponta achado semelhante ao observado na UNESP: um baixo nível de interação social entre as partes foi um dos aspectos que dificultou a TC, sendo uma das recomendações do estudo "ampliar o tempo que o pesquisador e o empresário dedicam a esse relacionamento".

Nessa direção, o estudo Segatto-Mendes e Mendes (2006) na PUC Paraná evidencia que as "conversações" e a "trocas de dados, propostas e ideias" entre coordenadores da empresa e professores foram estágios importantes para a concretização da parceria. Além disso, os achados apontam que a solução de conflitos relacionais que surgiram no decorrer do processo (em razão de diferenças de ideias e interesses entre a empresa e a universidade), se deu por meio de encontros e diálogos informais entre as partes, possibilitando “[...] o estabelecimento de maior confiança e o desenvolvimento de uma linguagem comum que muito facilitou a condução das atividades" (SEGATTO-MENDES; MENDES, 2006, p. 67).

Ainda no contexto brasileiro, a pesquisa de Closs et al. (2012), conduzido na PUC Rio Grande do Sul, reforça a importância da comunicação e das relações de confiança entre U-E no processo de TC. Achados do estudo sinalizam que quando a interação é caracterizada por diálogo e confiança, torna-se possível criar uma "linguagem comum” entre pesquisadores e funcionários da empresa, evitando conflitos relacionais que podem inviabilizar a transferência. Além disso, torna possível a transferência dos conhecimentos tácitos do 
“inventor” da tecnologia para a empresa facilitando a aplicação do conhecimento no contexto empresarial.

Além do relacionamento, outro fator que influencia na TC entre U-E é a distância cultural. A interação entre duas organizações com sistemas culturais distintos pode representar desafios em processos de TC, conforme sugerem estudos na área (BARBOLLA; CORREDERA, 2009; CLOSS et al., 2012; SCHOFIELD, 2013; DESIDÉRIO; ZILBER, 2014; SOUZA; SANTOYO, 2016). No caso da interação entre U-E, especificamente, Schofield (2013) aponta que as diferenças culturais estão relacionadas à (ao): missão de cada instituição; motivações para a transferência (o interesse da empresa em obter conhecimentos concretos pode chocar com o interesse de alguns pesquisadores em obter resultados acadêmicos); horizonte de tempo (algumas pesquisas demandam médio/longo prazo para gerar resultados, diferentemente da lógica de curto prazo do setor privado); linguagem dos pesquisadores e empresários. Tais diferenças podem se configurar como obstáculos na TC entre U-E na medida em que: (a) demanda esforços adicionais para o alinhamento de interesses na parceria (BARBOLLA; CORREDERA, 2009; SOUZA; SANTOYO, 2016); (b) requer uma aproximação intensa no decorrer da pesquisa para que pesquisadores e funcionários da empresa nivelem os seus conhecimentos (CLOSS et al., 2012); (c) exige interação pessoal frequente para facilitar o compartilhamento de conhecimentos tácitos dos professores para a empresa (CLOSS et al., 2012).

A pesquisa de Ipiranga, Fretas e Paiva (2010), por exemplo, realizada na Universidade Federal do Ceará, indica que o pesquisador, muitas vezes, tem a percepção de que o empresário não compreende a natureza da pesquisa científica, fazendo cobranças em relação a prazos e entregas que não podem ser atendidos pela universidade. Por outro lado, o empresário percebe uma desconexão entre a pesquisa universitária e as necessidades do setor produtivo e entende que há maior valorização, no contexto acadêmico, da pesquisa básica do que da pesquisa tecnológica aplicada.

O desalinhamento entre a pesquisa científica e as necessidades da indústria é, também, discutido no estudo de Closs et al. (2012), realizado na PUC Rio Grande do Sul. Os pesquisadores entrevistados reconhecem que, em geral, a academia “[...] ainda concebe a ciência aplicada como de segunda categoria", valorizando mais resultados acadêmicos (CLOSS et al., 2012, p. 69). Por outro lado, pontuam que a empresa brasileira não tem tradição de pesquisa e, portanto, não compreende a natureza incerta de tal atividade, esperando que o pesquisador alcance um resultado de fácil aplicação no contexto empresarial. 
Desse modo, os autores enfatizam a necessidade de se criar uma "linguagem comum" entre pesquisadores e empresários.

Nessa direção, o estudo de Segatto-Mendes e Mendes (2006) revela que, nos primeiros meses da parceria entre a PUC Paraná e a Electrolux, foram observadas dificuldades de comunicação e entendimento entre professores e o coordenador da empresa, em razão de diferenças de linguagem e de expectativas quanto aos prazos para o alcance dos resultados do projeto. Esse choque de interesses foi atenuado a partir da intensificação do relacionamento entre professores e o coordenador da empresa, permitindo, assim, o desenvolvimento de uma linguagem comum.

Além da distância cultural, estudos na área indicam que a TC entre U-E é afetada, também, por fatores de contexto universitário. Resultados de pesquisas que abordam a interação entre U-E no Brasil sinalizam que processos rígidos de TC, a ausência de políticas de incentivo e de setores de apoio a tal atividade representam barreiras à TC entre U-E (GARNICA; TORKOMIAN, 2009; SCHREIBER; PINHEIRO, 2011; CLOSS et al., 2012; DIAS; PORTO, 2014). Tais condições reduzem o interesse do professor em desenvolver parcerias com empresas e tornam mais moroso/ complexo o processo de tramitação e aprovação de projetos entre U-E no contexto universitário. Por outro lado, a TC entre U-E é favorecida em universidades que: (a) criam políticas de incentivo à TC, como portaria que institucionalize o apoio da reitoria, permissão para que os professores ganhem percentual do valor do projeto ao trazerem novas parcerias para a instituição; liberação de carga horária do docente; (b) flexibilizam as regras relacionadas à $\mathrm{TC}$, tornando o processo menos complexo e mais ágil; (c) criam setores de suporte a tal atividade.

O estudo de Garnica e Torkomian (2009), por exemplo, identificou que a morosidade dos trâmites internos relacionados à transferência tecnológica é um dos principais obstáculos ao desempenho do processo. O elevado tempo para a concretização da parceria e a falta de informações no decorrer do processo foram alguns dos desafios institucionais vivenciados pelas partes. Outro ponto importante abordado pelos autores é a necessidade de se estabelecer “[...]

retorno pecuniário para os inventores a título de estímulo" (GARNICA; TORKOMIAN, 2009, p. 634). Portanto, dentre outras implicações, o estudo de Garnica e Torkomian (2009) aponta como desafio das universidades brasileiras a promoção de uma reestruturação processual e de recursos humanos para a TC com o intuito de promover maior eficiência administrativa em tal processo, achado convergente com outras pesquisas brasileiras sobre o tema (DESIDÉRIO; ZILBER, 2014; DIAS; PORTO, 2014). 
O estudo de Schreiber e Pinheiro (2011), realizado em uma universidade privada de Porto Alegre, aponta alguns aspectos do contexto universitário que dificultaram a interação com o setor produtivo, dentre eles: a) a necessidade de investimento inicial (de horas) pelo professor para a atividade de prospecção de empresas; b) trâmite lento para a formalização das parcerias entre U-E; c) baixa colaboração dos setores administrativos que cuidam do processo; d) considerável burocracia interna. Tais condições prejudicam a TC ao reduzir o interesse do pesquisador em se envolver em tal atividade.

A questão da "burocracia” universitária, também, aparece na pesquisa de Araújo (2017). Dados do estudo evidenciam que o maior obstáculo que as empresas enfrentaram em projetos colaborativos com universidades foi a "burocracia" das instituições de ensino, seguido da situação de "não cumprimento de prazos". Em suas considerações finais, a autora sugere que as universidades criem uma nova política interna de TC em consonância com o marco legal da inovação de 2016.

Além das condições de nível organizacional, estudos na área indicam que a TC entre U-E é influenciada por características dos professores. Nessa direção, os estudos na área indicam que dois aspectos (embora não esgotem o assunto) parecem influenciar as atividades de TC: o know-how e a motivação dos docentes para a transferência. Universidades que detêm corpo docente com elevado know-how em sua área de conhecimento (boa reputação acadêmica, publicações de impacto, conhecimentos patenteados); que valorizam o desenvolvimento de pesquisas com empresas e são incentivados para tal têm maiores possibilidades de alcançar desempenho superior na TC. Quando existem essas condições, há, por um lado, maior interesse das empresas em estabelecer parcerias com a universidade e, por outro, maior motivação e capacidade do docente em desenvolver pesquisas colaborativas com empresas (GARNICA; TORKOMIAN, 2009; CLOSS et al., 2012; PERKMANN et al., 2013; SCHOFIELD, 2013; ARAÚJO; TEIXEIRA, 2014).

Nessa direção, o estudo de Closs et al. (2012), realizado na PUC Rio Grande do Sul, destacou o papel crítico do "professor inventor" na TC entre U-E. O envolvimento do professor desde o início da pesquisa até a efetiva transferência do conhecimento para a empresa é um ponto chave para o sucesso do processo, pois possibilita, ao docente, transferir os seus conhecimentos tácitos para funcionários da empresa, facilitando, assim, a aplicação do conhecimento no contexto empresarial.

O papel do comprometimento e envolvimento do professor é abordado, também, no estudo de Garnica e Torkomian (2009). Os autores constataram que determinadas características do pesquisador favorecem a TC entre U-E, especificamente: a) o seu nível de 
envolvimento no decorrer processo e a sua abertura para interagir com a empresa; b) o seu nível de qualificação técnica, o que amplia a confiança da empresa na capacidade do pesquisador; c) posse de tecnologia com potencial de aplicabilidade no mercado.

A pesquisa de Desiderio e Zilber (2014), por sua vez, enfatiza o impacto da "visão mercadológica" do pesquisador na TC, apontando que muitos pesquisadores não tem uma visão de mercado que possibilita aos mesmos entender que determinadas tecnologias têm potencial de comercialização. Os autores pontuam, ainda, que outra dificuldade está relacionada à ênfase do professor em desenvolver conhecimentos de natureza mais acadêmica se dedicando pouco a pesquisas aplicadas de interesse do setor produtivo.

Diante da discussão teórica apresentada, o Quadro 1 reúne os fatores ora discutidos.

\section{Quadro 1- Fatores Críticos da TC entre U-E}

\begin{tabular}{|l|l|l|}
\hline \multicolumn{1}{|c|}{ Fator } & \multicolumn{1}{|c|}{ Descrição } & \multicolumn{1}{c|}{ Autores } \\
\hline $\begin{array}{l}\text { Relacionamento } \\
\text { entre U-E }\end{array}$ & $\begin{array}{l}\text { Nível de conexão social, } \\
\text { comunicação e confiança }\end{array}$ & $\begin{array}{l}\text { (SEGATTO-MENDES; MENDES; 2016; ARAÚJO; } \\
\text { TEIXEIRA, 2014; SCHOFIELD, 2013; CLOSS et al. 2012; } \\
\text { BARBOLLA; CORREDERA, 2009; GARNICA; } \\
\text { TORKOMIAN, 2009; SHERWOOD; COVIN, 2008) }\end{array}$ \\
\hline Distância cultural & $\begin{array}{l}\text { Diferenças de linguagem e } \\
\text { interesses }\end{array}$ & $\begin{array}{l}\text { (SIEGEL et al. 2004; SEGATTO-MENDES; MENDES, } \\
\text { 2006; IPIRANGA; FREITAS; PAIVA, 2010; CLOSS et al., } \\
\text { 2012; SCHOFIELD, 2013; SOUZA; SANTOYO, 2016) }\end{array}$ \\
\hline $\begin{array}{l}\text { Condições } \\
\text { Universitárias }\end{array}$ & $\begin{array}{l}\text { Políticas de incentivo à TC } \\
\text { e flexibilidade dos } \\
\text { processos de TC }\end{array}$ & $\begin{array}{l}\text { (DIAS; PORTO, 2014; PERKMANN et al., 2013; CLOSS et } \\
\text { al., 2012; SCHREIBER; PINHEIRO, 2011; GARNICA, } \\
\text { TORKOMIAN, 2009) }\end{array}$ \\
\hline $\begin{array}{l}\text { Características } \\
\text { dos professores }\end{array}$ & $\begin{array}{l}\text { Know-how do professor e a } \\
\text { sua motivação para a TC }\end{array}$ & $\begin{array}{l}\text { (BEKKERS; BODAS; FREITAS, 2008; GARNICA; } \\
\text { TORKOMIAN, 2009; CLOSS et al., 2012; PERKMANN et } \\
\text { al., 2013; SCHOFIELD, 2013; HU et al., 2016) }\end{array}$ \\
\hline
\end{tabular}

Fonte: elaborado pelos autores.

Discutidas as bases teóricas, a próxima seção apresenta o método de pesquisa.

\section{METODOLOGIA}

Para o alcance do objetivo proposto, foi realizada pesquisa qualitativa com base no método de estudo de caso instrumental (STAKE, 2005; YIN, 2010), conforme detalha o Quadro 2. 


\section{Quadro 2 - Método de pesquisa}

\begin{tabular}{|c|c|}
\hline Item & Descrição \\
\hline Estratégia & Qualitativa \\
\hline Método & Estudo de caso instrumental \\
\hline Contexto & Universidade Federal de Minas Gerais \\
\hline $\begin{array}{l}\text { Unidades de } \\
\text { observação }\end{array}$ & $\begin{array}{l}\text { Dois projetos desenvolvidos entre uma universidade pública brasileira e empresas } \\
\text { parceiras, um na área de meio-ambiente e um projeto interdisciplinar }\end{array}$ \\
\hline Coleta de dados & 14 entrevistas em profundidade e documentos secundários \\
\hline $\begin{array}{c}\text { Sujeitos } \\
\text { entrevistados }\end{array}$ & $\begin{array}{l}\text { Universidade: coordenador da pesquisa; professores participantes; alunos envolvidos; } \\
\text { equipe da área de transferência e inovação tecnológica }\end{array}$ \\
\hline Análise de dados & Análise de conteúdo, com o apoio do Software $N$-vivo \\
\hline
\end{tabular}

Fonte: elaborado pelos autores.

Em relação ao método de pesquisa, entende-se que o estudo de caso foi adequado para a análise do fenômeno da TC entre U-E, considerando que: a) o objetivo da pesquisa foi o de analisar "como" fatores críticos influenciaram na TC entre a Universidade e empresas; b) a TC entre U-E é um tema que tem ganhado destaque na literatura na década de 2000 e, por ser contemporâneo, há questões que demandam maior aprofundamento.

Segundo Stake (2005), o pesquisador pode optar por três tipos de estudos de caso: intrínseco, coletivo e instrumental. O primeiro é adequado quando o pesquisador pretende conhecer determinado caso em profundidade (um caso específico), atendo-se a toda a sua particularidade e sem preocupação com o desenvolvimento de teoria. Já o caso coletivo é utilizado para estudar características de um "universo" de casos, com o intuito de compreender características comuns e divergentes entre eles. Por fim, o caso instrumental é recomendado quando o pesquisador tem o propósito principal de fornecer insigths relevantes sobre determinado problema. O caso desempenha papel de apoio com o intuito de facilitar uma compreensão acerca da questão investigada.

Tomando como referência a tipologia de Stake (2005), foi adotado o estudo de caso instrumental, pois o objetivo do estudo foi o de fornecer insigths sobre os fatores críticos da TC entre U-E, sendo a Universidade o contexto "de apoio" que possibilitou uma compreensão da questão teórica proposta. Nessa direção, entende-se que o caso escolhido é adequado, pois a Universidade: a) assumiu, em 2016, a liderança no ranking do Instituto Nacional da Propriedade Industrial (INPI) como a instituição brasileira que mais fez pedidos de patentes de invenções no país; b) criou setor de apoio à transferência e inovação tecnológica, em 1997, antes da Lei de Inovação de 2004 que regulamentou sobre a 
obrigatoriedade das universidades terem uma estrutura dessa natureza (BRASIL, 2017). Tais dados sinalizam que a universidade investigada contempla requisitos que a tornam uma instituição relevante em termos de patente, inovação e transferência.

Para a seleção dos projetos, foi feito contato com a CTIT, setor vinculado à Próreitoria de Pesquisa, criado em 1997, que atua na gestão do conhecimento gerado na Universidade. Com base nesses dados, foram identificados professores com experiência no desenvolvimento de pesquisa com empresas e com bom desempenho em indicadores acadêmicos (tais como publicações, patentes, produtos tecnológicos).

Quanto à coleta de dados, foram utilizadas entrevistas semiestruturadas e documentos secundários. O roteiro de entrevista foi elaborado com base nos fatores discutidos na revisão teórica da pesquisa. Foram conduzidas 14 entrevistas (sendo 12 atores da universidade e 2 das empresas parceiras), no período de setembro/2017 a dezembro/2017, com os principais agentes envolvidos nos projetos.

As entrevistas foram gravadas e transcritas para facilitar o tratamento das informações no Software $N$-vivo. Complementarmente aos dados primários, a pesquisa documental contemplou análise de diversos documentos, dentre eles: convênios de cooperação; relatórios de pesquisa; relatórios de patentes do INPI; portarias da Universidade relacionadas à inovação e TC; planilhas e atas de reunião disponibilizados pela CTIT.

Quanto ao tratamento dos dados, foi adotada a técnica de análise de conteúdo (por categoria) que consiste na análise de materiais textuais, envolvendo atividades de organização, categorização, interpretação e descrição do conteúdo das informações. O pesquisador busca compreender profundamente o texto, examinar suas várias dimensões e construir inferências a partir dele (BAUER; GASKELL, 2002). Para facilitar esse exame, foram tomadas como referência as seguintes categorias teóricas: relacionamento entre U-E; distância cultural; condições universitárias; características dos professores.

Discutidas as escolhas metodológicas, o próximo tópico apresenta a análise dos dados.

\section{APRESENTAÇÃO E ANÁLISE DOS DADOS}

\subsection{PROJETO INTERDISCIPLINAR}

O projeto em análise é fruto de parceria desenvolvida entre vários departamentos da Universidade e uma empresa privada do setor de cimentos com o objetivo de aprimorar processos baseados em nanotubos de carbono, desenvolvidos por professores da Universidade, em produtos cimentícios da empresa parceira. 


\section{Quadro 3 - Descrição do Projeto 1}

\begin{tabular}{|l|l|}
\hline \multicolumn{1}{|c|}{ Item } & \multicolumn{1}{c|}{ Descrição } \\
\hline Natureza & Convênio de Cooperação Técnica \\
\hline Prazo & 18 meses a contar de dezembro de 2009 \\
\hline Título do projeto & $\begin{array}{l}\text { Processo de síntese contínua e em larga escala de nanotubos de carbono sobre o clínquer } \\
\text { de cimentos e produção nanoestruturada }\end{array}$ \\
\hline $\begin{array}{l}\text { Resultados } \\
\text { Obtidos }\end{array}$ & $\begin{array}{l}\text { - Melhoria de processos } \\
\text { - Pelhoria de produto: Cimento Nanoestruturado }\end{array}$ \\
\hline
\end{tabular}

Fonte: elaborado pelos autores.

Com relação aos fatores considerados críticos para o desempenho do Projeto 1, os dados empíricos apontam para a importância de dois fatores: (a) professores com know-how e conhecimentos inovadores na área de pesquisa (Entrevistas 1, 2, 3, 4, 5); (b) o relacionamento e o alinhamento estabelecidos entre a equipe da Universidade e da empresa de cimentos (Entrevistas 1, 2, 3, 4). Tais achados corroboram a importância de dois fatores contemplados na revisão teórica de pesquisa: know-how dos professores (CLOSS et al., 2012; PERKMANN et al., 2013; SCHOFIELD, 2013) e o relacionamento entre U-E (BARBOLLA; CORREDERA, 2009; CLOSS et al. 2012).

Em relação ao primeiro aspecto, os entrevistados revelam que a existência de professores com know-how e conhecimentos inovadores na área de "nanotubos de carbono" foram decisivos no Projeto 1 ao ampliar o interesse da empresa para cooperar com a Universidade (Entrevistas 1, 2, 3, 4, 5). Ao considerar a parceria atrativa, o funcionário e o gerente da empresa se esforçaram para interagir com os pesquisadores e se envolveram em várias etapas do projeto, fornecendo feedbacks sobre a evolução da pesquisa.

Além disso, os dados relevam que a expertise dos professores da Universidade na área aumentou a confiança da empresa no trabalho da instituição, gerando maior respeitabilidade na parceria (Entrevistas 1, 2, 3, 4, 5). Tais análises reforçam resultados de estudos, como o de Bekkers et al. (2008) e o de Garnica e Torkomian (2009), de que um know-how comprovado em determinada área de conhecimento torna a parceria mais atrativa do ponto de vista da empresa, bem como o de $\mathrm{Hu}$ et al. (2016), que enfatiza a importância da "reputação do professor".

Outro fator considerado crítico pelos entrevistados foi o relacionamento e o alinhamento construído entre a Universidade e a empresa de cimentos (Entrevistas 1, 2, 3, 4). 
Em linhas gerais, tal alinhamento foi possível, pois as partes buscaram trabalhar de forma próxima e estabeleceram troca constante de informações no decorrer do projeto. Consequentemente, a empresa de cimentos pôde direcionar o desenvolvimento da pesquisa, permitindo, por um lado, que o projeto atendesse às necessidades da empresa e, por outro, que o conhecimento universitário fosse testado e aprimorado, convergente com a expectativa dos professores.

Com relação ao nível de confiança entre a Universidade e a empresa de cimentos, os dados empíricos indicam que o contato frequente e o trabalho conjunto desenvolvido entre as partes contribuíram para a criação de um ambiente de confiança (Entrevistas 1, 2, 3), o que na visão dos sujeitos entrevistados, é um aspecto crucial em projetos que envolvem questões estratégicas para as empresas: "A relação U-E, ela é baseada na confiança. Por quê? Porque o lado deles lá é muito competitivo. Então eles precisam do sigilo para garantir" (Entrevista 1). Portanto, na ausência de confiança, universidade e empresa podem ter receio de compartilhar informações relevantes, (ARAÚJO; TEIXEIRA, 2014), o que não foi constatado no Projeto 1.

Além dos fatores críticos, a pesquisa empírica contemplou, também, questões relacionadas à influência da "distância cultural" na TC. No Projeto 1, parte dos entrevistados pontua, primeiramente, que ainda existe "preconceito" em relação à interação entre U-E no Brasil, o que já se configura como um desafio antes mesmo de uma parceria ser concretizada. No Projeto 1, especificamente, os dados sinalizam que a distância cultural que, em geral, existe entre o ambiente acadêmico e o empresarial foi atenuada pelo relacionamento estabelecido entre a Universidade e a empresa de cimentos (Entrevistas 1, 3, 4, 5). Ou seja, o ambiente de interação e comunicação criado entre as partes permitiu um trabalho conjunto e o alinhamento de interesses, evitando alguns conflitos que são comuns na interação entre U-E: "A visão do mercado é muito diferente. Tem que ter um meio do caminho, e esse meio do caminho só vai acontecer se for com o trabalho junto" (Entrevista 3).

Além das diferenças de prazos, os entrevistados comentam sobre outra fonte de conflito comum entre academia-empresas: "a natureza básica ou aplicada da pesquisa", a qual não foi constada no Projeto 1 em razão do perfil dos professores envolvidos (Entrevistas 1, 2, 3, 5). Ou seja, há indícios de que esses docentes sempre buscaram desenvolver pesquisa aplicada ao longo de suas trajetórias acadêmicas. Portanto, conforme aponta Barbolla e Corredera (2009), quando o projeto envolve professores que valorizam o desenvolvimento de pesquisa aplicada, há maior alinhamento entre as expectativas dos professores e dos empresários, contribuindo, assim, para minimizar potenciais conflitos na interação entre U-E. 
A pesquisa empírica contemplou, também, questões relacionadas à influência das condições universitárias na TC. Ao serem questionados sobre as condições organizacionais da Universidade, é unânime a percepção de que existem desafios internos em relação ao processo de TC para o setor produtivo (Entrevista 1, 2, 3, 4, 5). Os entrevistados reconhecem que a Universidade é uma das instituições brasileiras que mais gera patente e que muitas melhorias foram alcançadas com o apoio da CTIT, mas salientam que há ainda muito o que ser feito para que a instituição seja um ambiente de fomento às atividades de TT.

Quanto às "políticas de incentivo", os entrevistados afirmam que o apoio institucional para o professor se engajar em projetos com empresas é muito "tímido", senão inexistente. Não há políticas sistematizadas de incentivo à $\mathrm{TC}$ e, consequentemente, tais parcerias dependem de iniciativas pessoais.

Quanto ao nível de flexibilidade dos processos de TC, os entrevistados do Projeto 1 revelam que enfrentam elevada "burocracia" para a formalização de parcerias com empresas na Universidade, como o excesso de instâncias hierárquicas para tramitação, o elevado tempo para aprovação, o "conservadorismo" e a "rigidez" da área jurídica da universidade (Entrevista 1, 2, 3, 5), achados convergentes com pesquisas realizadas em outras universidades brasileiras (CLOSS et al., 2012; GARNICA; TORKOMIAN, 2009).

Quanto aos setores de apoio à TC, os entrevistados reforçam o importante papel da área de transferência tecnológica da Universidade na interface com o setor produtivo e na orientação das regras/trâmites relacionadas à TC para o mercado. Todavia, alguns entrevistados sinalizam que é necessário maior "amadurecimento" das iniciativas relacionadas à TC (Entrevistas 3,4$)$.

Além das condições organizacionais da universidade, o roteiro de entrevistas contemplou, também, questões relacionadas à influência das características dos pesquisadores na TC. Os dados empíricos do Projeto 1 sinalizam que o know-how dos professores, bem como a sua motivação para a transferência foram considerados aspectos relevantes para o alcance dos resultados pretendidos (Entrevistas 1, 2, 3, 4, 5), achados convergentes com estudos prévios que destacam o papel do "inventor" da tecnologia na interação entre U-E (GARNICA; TORKOMIAN, 2009; CLOSS et al., 2012; HU et al., 2016).

Os dados indicam que a motivação dos professores participantes do Projeto 1 foi um fator importante para o alcance dos resultados (Entrevistas 1, 3, 4, 5), pois são pesquisadores que buscam desenvolver pesquisas com foco em aplicações e que possuem experiências de colaboração com o setor produtivo. Esse perfil favorece a TC para as empresas, pois há maior abertura e "proatividade" dos mesmos para colaborar com empresas, o que nem sempre é 
uma realidade no ambiente universitário. Segundo Desiderio e Zilber (2014), docentes que não possuem "visão de pesquisa aplicada" reduzem as possibilidades de interação entre U-E, dado que o setor produtivo tem mais interesse em pesquisas aplicáveis às rotinas da organização.

Analisando os achados do Projeto 1, observa-se que o seu desempenho foi favorecido pelo modelo de relacionamento estabelecido entre a equipe da Universidade e da empresa de cimentos; pelo Know-how dos professores e posse de tecnologia inovadora de interesse do mercado. Quanto às diferenças culturais, por mais que elas tenham emergido em algumas situações no decorrer do Projeto 1, há indícios de que foram atenuadas pelo ambiente cooperativo e de confiança criado entre as partes. Por outro lado, aspectos do contexto institucional (como a "burocracia" e a ausência de incentivos à TC na Universidade) podem, na visão dos entrevistados, ter representado desafios na parceria investigada.

Dando sequência à análise dos casos, a próxima seção apresenta o projeto na área de Meio Ambiente.

\subsection{PROJETO NA ÁREA DE MEIO AMBIENTE}

O caso em análise é fruto de parceria desenvolvida entre a Universidade através do Departamento de Química e uma empresa mineira do segmento de consultoria em tratamento de água, conforme Quadro 4.

\section{Quadro 4 - Descrição do Projeto 2}

\begin{tabular}{|l|l|}
\hline \multicolumn{1}{|c|}{ Item } & \multicolumn{1}{c|}{ Descrição } \\
\hline Natureza da parceria & Contrato de licenciamento de tecnologia patenteada \\
\hline Objeto & $\begin{array}{l}\text { Concessão de licença para a empresa explorar o "supressor de poeira à base de } \\
\text { glicerina", tecnologia voltada para produção de material polimérico eficiente que } \\
\text { minimiza os impactos da poeira gerada na indústria }\end{array}$ \\
\hline Resultado Obtido & $\begin{array}{l}\text { Licença de tecnologia } \\
\text { Melhoria de produto: supressor de poeira à base de glicerina }\end{array}$ \\
\hline
\end{tabular}

Fonte: elaborado pelos autores

Com relação aos fatores considerados críticos para o desempenho do Projeto 2, há consenso de que ele foi favorecido pelas características do professor, tanto em termos do seu perfil e da sua motivação para a transferência, quanto em relação à posse de tecnologia de 
interesse do mercado (Entrevistas 6, 7, 8 e 9). Primeiramente, os resultados indicam que, para a parceria acontecer, foi fundamental que o professor tivesse interesse em transformar o conhecimento desenvolvido em escala laboratorial em aplicações úteis à realidade das empresas (Entrevistas 6, 7, 8 e 9). Ou seja, requer um perfil de professor que deseja ir além das produções acadêmicas, que segundo Desidério e Zilber (2014), são docentes com visão de mercado.

Além do perfil e da motivação do professor, outro aspecto considerado crítico para o desempenho do Projeto 2 é o know-how do docente e a posse de tecnologia de interesse de mercado (Entrevistas 6, 9, 10). O coordenador do projeto tem background reconhecido na área de meio-ambiente, possui 28 patentes registradas no INPI e tinha desenvolvido tecnologia inovadora, o que ampliou o interesse da empresa em colaborar com a universidade: "Era uma tecnologia à base de glicerina, totalmente sustentável e de uma fonte renovável. Era uma inovação" (Entrevista 10).

Além dos fatores críticos, o roteiro de pesquisa contemplou, também, questões relacionadas à influência do relacionamento na TC entre U-E. No caso do Projeto 2, há consenso entre os entrevistados de que o modelo de relacionamento estabelecido entre a Universidade e a empresa favoreceu o alcance dos resultados do projeto (situação semelhante à observada no Projeto 1). Os achados indicam que houve um trabalho de construção conjunta e amplo envolvimento das partes na TC, permitindo a construção de um ambiente de confiança (Entrevistas 6, 7, 8, 10).

Quanto ao nível de “conexão social”, constatou-se um elevado nível de proximidade entre a equipe da universidade e da empresa mesmo após o licenciamento do supressor de poeira, possibilitando a resolução conjunta de problemas que emergiram no decorrer da pesquisa. Segundo Garnica e Torkomian (2009), essa interação entre U-E é fundamental, pois um acompanhamento pouco sistemático dos pesquisadores na fase de aplicação da tecnologia na empresa representa um dos desafios enfrentados na TC.

Com relação ao nível de confiança, os entrevistados pontuam que na sua ausência, o trabalho cooperativo pode ficar comprometido: "Sem confiança, eu não te passaria informações confidenciais, eu não proporia para você um acordo" (Entrevista 7). Portanto, na relação empresário-pesquisador "confiança é essencial, tanto do ponto de vista técnico quanto do ponto de vista ético" (Entrevista 7).

Além dos fatores considerados críticos, o roteiro de entrevista contemplou, também, questões relacionadas à influência da distância cultural na TC. Semelhante ao constatado no Projeto 1, os entrevistados do Projeto 2 reconhecem que academia-indústria são dois 
ambientes distintos e que tais diferenças emergiram em algumas situações no decorrer do projeto (Entrevistas 6, 7, 8, 9, 10). Por exemplo, o professor tinha expectativa de realizar os testes considerando os parâmetros de rigor científico, o que levaria mais tempo para a finalização do projeto e, por outro lado, o diretor da empresa esperava por resultados "imediatos" (Entrevista 9). Todavia, os dados revelam como o trabalho conjunto permitiu que os interesses de ambas as partes fossem, de alguma forma, equilibrados, atenuando a percepção de distância cultural entre a Universidade e a empresa parceira.

Um dado interessante que emergiu nas entrevistas (e que foi pouco explorado na literatura sobre o tema), é que um pesquisador "com visão mercadológica" pode contribuir para minimizar a percepção de distância cultural entre U-E, na medida em que, neste caso, as diferenças de linguagem e interesses entre academia-indústria tendem a ser menores.

Além dos fatores de natureza relacional, a pesquisa contemplou questões relacionadas à influência das condições organizacionais da Universidade na TC. Em linhas gerais, os entrevistados reconhecem que a Universidade "está à frente" de muitas universidades brasileiras quando o assunto é pesquisa e inovação e que, nos últimos anos, a área de transferência tecnológica tem catalisado esforços para melhorar a interface da universidade com o setor produtivo (Entrevistas 6, 7, 8, 9). Na visão dos entrevistados, o setor fornece as orientações necessárias para a formalização de parcerias com empresas facilitando, assim, o diálogo entre U-E: "A Universidade é um exemplo para o Brasil. A área de transferência tecnológica realmente facilita a conversa com indústrias (Entrevista 7).

Se a área de transferência tecnológica é considerada um setor de apoio institucional, os entrevistados do Projeto 2 criticam o papel da Procuradoria Jurídica da Universidade, enfatizando que o setor possui uma visão conservadora e contrária, inclusive, ao novo marco legal da inovação, que torna mais flexível o processo de TC: "De entrave tem a PJ, que, além de ser muito lenta, é conservadora. Questões que já estão resolvidas na legislação, ainda travam aqui" (Entrevista 8). Portanto, a questão da "burocracia" é um tema recorrente em pesquisas nacionais que abordam a interação entre U-E no país, como as de Garnica e Torkomian (2009) e Araújo (2017).

Quanto às "políticas de incentivo", os entrevistados reconhecem que algumas iniciativas têm sido empreendidas na Universidade para institucionalizar uma política de inovação na instituição. Em 2016, por exemplo, a Reitoria criou uma comissão para estudar a Lei 13.243/2016 e propor a sua normatização na universidade, representando um passo importante para difundir uma cultura de interação entre U-E na Universidade. 
Analisando os achados do Projeto 2, observa-se que o seu desempenho foi favorecido pelo relacionamento estabelecido entre a equipe da Universidade e da empresa; pela postura do professor-coordenador e pela posse de tecnologia de interesse do mercado. Quanto às diferenças culturais, por mais que elas tenham emergido no decorrer do Projeto 2, há indícios de que foram atenuadas pelo ambiente cooperativo criado entre as partes, bem como pelo perfil do professor-coordenador. Por outro lado, um desafio mencionado nessa parceria foi a “rigidez" dos processos jurídicos de TC da Universidade.

\section{CONSIDERAÇÕES FINAIS}

Tomando como referência o objetivo do estudo, constata-se que dois fatores foram considerados decisivos para o alcance dos resultados dos projetos analisados, quais sejam: as características do professor, sobretudo o seu know-how e a sua postura/motivação na TC; e o relacionamento entre a Universidade e a empresa parceira.

Tais achados indicam, por um lado, que a abertura da empresa para colaborar com a universidade depende de professores com know-how reconhecido na sua área, seja através dos conhecimentos que o docente obteve ao longo da sua trajetória acadêmica, seja pela posse de tecnologia inovadora com potencial de aplicabilidade no setor produtivo. Nesse sentido, as empresas vêm buscando um "nome" ou uma "tecnologia potencial", reforçando a importância da reputação do professor, conforme sugerem Hu et al. (2016). Tal condição, por um lado, torna a parceria atrativa para a empresa, e por outro, aumenta a confiança dos empresários no trabalho da instituição, corroborando discussões feitas em estudos prévios (GARNICA; TORKOMIAN, 2009; ARAÚJO; TEIXEIRA, 2014).

Essa discussão tem relação com um conceito bastante discutido em estudos sobre TC interorganizacional, mas pouco abordado nas pesquisas específicas sobre TC entre U-E: a atratividade da fonte (PÉREZ-NORDTVEDT et al., 2008). Tomando como referência essa variável, pode-se inferir que quando a empresa fonte (a universidade) é percebida como atrativa, isto é, detentora de conhecimentos relevantes e com experiência em projetos de transferência, haverá maior predisposição da empresa para participar do processo, bem como para aplicar o conhecimento nas rotinas organizacionais. Portanto, o nível de abertura da empresa para interagir com a universidade pode estar associada à atratividade da fonte, que, por sua vez, está relacionada ao know-how dos professores.

Quanto à motivação do professor, os resultados da pesquisa levam ao entendimento de que a TC depende de um perfil de pesquisador que deseja ir além das produções acadêmicas e 
que enxerga valor na disseminação de seus conhecimentos para o mercado. Quando o professor tem esse entendimento é mais provável que ele esteja aberto para colaborar com empresas. Caso contrário, ele pode até deter um conhecimento relevante, mas não se interessar em disponibilizá-lo para as empresas, inviabilizando a realização de um projeto de TC. Portanto, a motivação do professor é um elemento que deve ser considerado em modelos de TC.

Os resultados indicam, também, que a TC entre U-E depende da construção de um relacionamento baseado em conexão social, comunicação e confiança. Nos Projetos 1 e 2 foi possível observar um ambiente relacional com essas características o que favoreceu o alcance dos resultados pretendidos, na medida em que: contribuiu para minimizar a percepção de distância cultural entre a Universidade e empresas parceiras; possibilitou um acompanhamento conjunto das duas equipes no decorrer do projeto; intensificou o vínculo entre professores e funcionários das empresas ampliando o comprometimento das partes com o projeto.

Quanto à sua contribuição teórica, o estudo realizado avança ao apontar fatores críticos da transferência de conhecimento entre U-E, contribuindo para elucidar como fatores de contexto relacional e universitário influenciam no desempenho desse processo, um tópico de pesquisa considerado contemporâneo e relevante (FELIU; RODRÍGUES, 2017). Em termos práticos, o estudo destaca a importância da interação entre U-E disseminando ideias que possam contribuir, de alguma forma, para o fortalecimento da cultura de cooperação entre o ambiente acadêmico e o empresarial no país.

\section{REFERÊNCIAS}

ARAUJO, Carina; TEIXEIRA, Aurora. Determinants of International Technology Transfer: an Empirical Analysis of the Enterprise Europe Network. Journal of Technology

Management \& Innovation, Santiago, v. 9, n. 3, p. 120-134, 2014.

ARAUJO, Janaína Coelho. A contribuição da comunicação nos processos de transferência de tecnologias nas universidades: o caso da UFMG. Dissertação. Mestrado Profissional em Inovação Tecnológica e Propriedade Intelectual, Universidade Federal de Minas Gerais, Belo Horizonte, 2017.

BARBOLLA, Ana Bernardos; CORREDERA, José Casar. Critical factors for success in university-industry research projects. Technology Management and Strategic Analysis, v. 21, n.5, p. 599-616, 2009. 
BAUER, Martin W.; GASKELL, George. Pesquisa Qualitativa com Texto, Imagem e Som: um manual prático. 2.ed. Petrópolis: Vozes, 2002, 516 p.

BEKKERS, Rudy; BODAS FREITAS, Isabel Maria. M. Analysing knowledge transfer channels between universities and industry: to what degree do sectors also matter? Research Policy, v. 37, n. 10, p. 1837-1853, 2008.

BONOMA, Thomas V. Case research in Marketing: Opportunities, Problems and a Process. Journal of Marketing Research. v. XXII, 1985.

BRASIL. Lei n.10.973, de 2 de dezembro de 2004. Dispõe sobre incentivos à inovação e à pesquisa científica e tecnológica no ambiente produtivo e dá outras providências. Disponível em: $<$ http://www.planalto.gov.br/ccivil_03/_ato2004-2006/2004/Lei/L10.973.htm>. Acesso em 10 de outubro de 2017.

CHESBROUGH, H. The Future of Open Innovation. Journal Research-Technology Management, v. 60, n. 1, 2017, p. 35-38, 2017.

CLOSS, Lisiane; FERREIRA, Gabriela; SAMPAIO, Cláudio; PERIN, Marcelo. Intervenientes na transferência de tecnologia universidade-empresa: o caso PUCRS. Revista de Administração Contemporânea, v. 16, n. 1, p. 59-78, 2012.

CLOSS, Lisiane et al. What Motivates Brazilian Academic Researchers to Transfer Technology? Journal of Technology Management \& Innovation, v. 8, n. 4, p. 79-90, 2013. DESIDÉRIO, Paulo Henrique Martins; ZILBER, Moisés Ari. Barreiras no processo de transferência tecnológica entre agências de inovação e empresas: observações em universidades públicas e privadas. Revista Gestão \& Tecnologia, v. 14, n. 2, p. 99-124, 2014.

DIAS, Alexandre Aparecido; PORTO, Geciane Silveira. Gestão de transferência de tecnologia na inova Unicamp. Revista de Administração Contemporânea, Curitiba, v. 17, n. 3, p. 263-284, 2013.

ETZKOWITZ, Henry; LEYDESDORFF, Loet. The triple helix-university industrygovernment relations: a laboratory for knowledge based economic development. European Association Study Science and Technology Review, v. 14, n. 1, p. 14-19, 1997. GARNICA, Leonardo Augusto; TORKOMIAN, Ana Lúcia Vitale. Gestão de tecnologia em universidades: uma análise do patenteamento e dos fatores de dificuldade e de apoio à transferência de tecnologia no Estado de São Paulo. Gestão e Produção, v. 16, n. 4, p. 624 $638,2009$. 
HU, M.; HUNG, S.; LO, H, TSENG, Yung-Ching. Determinants of university-industry research collaborations in Taiwan: The case of the National Tsing Hua University. Research evaluation, v. 25, n .2, p. 121-135, 2016.

IPIRANGA, Ana Silva et al. O empreendedorismo acadêmico no contexto da interação universidade- empresa -governo. Cadernos Ebape, v. 8, n.4, p. 676-693, 2010.

MURMANN, J. P. The co-development of industrial sectors and academic disciplines.

Science and Public Policy, v. 40, n. 2, p. 229-246, 2013.

PÉREZ-NORDTVEDT et al. Effectiveness and Efficiency of Cross-Border Knowledge Transfer: An Empirical Examination. Journal of Management Studies, v. 45, n. 4, p. 714$744,2008$.

PERKMANN, Markus, et al. Academic engagement and commercialisation: A review of the literature on university-industry relations. Research Policy, v. 42, n. 2, p. 423-442, 2013. SANTORO, Michael; BIERLY, Paul. Facilitators of knowledge transfer in university-industry collaborations: a knowledge-based perspective. IEEE Transactions on Engineering Management, v. 53 n. 4, p. 495-507, 2006.

SCHOFIELD, Tatiana. Critical success factors for knowledge transfer collaborations between university and industry. Journal of Research Administration, v. 44, n. 2, p. 38-56, 2013. SCHREIBER, D.; PINHEIRO, I. A. A influência da cultura organizacional de uma IES no processo de interação universidade - empresa. Revista Alcance, v. 18, n. 3, p. 258-270, 2011. SEGATTO-MENDES, Andréa Paula; MENDES, Nathan. Cooperação tecnológica universidade-empresa para eficiência energética: um estudo de caso. Revista de Administração Contemporânea, Curitiba , v. 10, n. spe, p. 53-75, 2006 . SHERWOOD, Arthur; COVIN, Jeffrey. Knowledge acquisition in university-industry alliances: an empirical investigation from a learning theory perspective. Journal of Product Innovation Management, v. 25, n. 2, p. 162-79, 2008.

SIEGEL, Donald et al. Toward a model of the effective transfer of scientific knowledge from academicians to practitioners: Qualitative evidence from the commercialization of university technologies. Journal of Engineering and Technology Management, v. 21, n. 1-2, p. 11542, 2004.

STAKE, R. Case Studies. In: DENZIN, N.; LINCOLN, T. Handbook of Qualitative Research. London: Sage, p. 134-164, 2005.

STAL, Eva; FUJINO, Asa. As relações universidade-empresa no Brasil sob a ótica da Lei de Inovação. Revista de Administração e Inovação, v. 2, n. 1, p. 5-19, 2005. 
STAL, Eva; FUJINO, Asa. The evolution of universities' relations with the business sector in Brazil: What national publications between 1980 and 2012 reveal. Revista de

Administração (São Paulo), v. 51, n. 1, p. 72-86, Mar. 2016.

YIN, Robert. Estudo de caso: planejamento e métodos. 4. ed. Porto Alegre: Bookman, xvii, 248 p., 2010. 